\title{
ANC System for Noisy Speech
}

\author{
Sivadasan Kottayi ${ }^{1}$ and Narayanan N.K. ${ }^{2}$ \\ ${ }^{1}$ Electronics \& Instrumentation Engineering Technology Department, \\ Yanbu Industrial College, Yanbu, Saudi Arabia. \\ Email: sivadasan.kottayi@gmail.com \\ ${ }^{2}$ Information Technology Department, Kannur University, Kannur 670567, India. \\ Email: csircerediffmail.com
}

\begin{abstract}
The paper explains the effectiveness of Active Noise Control (ANC) System based on adaptive filter for reducing noise from a noisy speech. ANC generally reduces low frequency noise and creates a quiet zone. In this paper a feedforward ANC is used for reducing low frequency noise from a noisy speech signal for improving speech quality. Secondary path transfer function has been compensated using FXNLMS (Filtered-X NLMS) algorithm. The proposed system is analyzed and simulated on a digital computer. The performance of the system is evaluated by measuring noise power reduction in the noisy speech by retaining the intelligibility of the speech signal. Simulation results reveal that the method can reduce noise power to a level of $-11.6 \mathrm{~dB}$.
\end{abstract}

\section{Keywords}

Active noise control, Active noise cancellation, Adaptive systems, Speech processing, Digital Signal Processing Application.

\section{Introduction}

There are two types of noise; high frequency noise and low frequency noise. High frequency noise can be reduced by traditional passive techniques, which employ heavy barriers to block the transmission of sound, and also use certain acoustic materials to absorb sound energy. However, such techniques are found less effective at low frequency $(<500 \mathrm{~Hz})$. Low frequency noise at market places, buses, trains, offices (due to the operation of air conditioners and fans), Industries (due to the operation of various machines) etc. often corrupt speech signals. While receiving phone call in a mobile phone at a noisy market place or inside a bus or train, the message coming through the phone loses its intelligibility and also the surrounding noise propagates to the distant caller. Conventional passive techniques cannot be applied in such situations, but active noise control (ANC) [1] - [5] can be used.

\section{Technique of Active Noise Control}

ANC is based on the destructive interference between two sound fields; one field is the original or primary sound source and the other from a secondary sound source generated by an electronic system having same frequency of primary sound but $180^{\circ}$ out of phase, which is propagated through a loudspeaker to interfere with the primary sound so that both get cancelled by themselves. Generally two control strategies [1,2 ] namely feed-forward ANC control as well as 
feedback ANC control are popular. In this paper, primary noise is considered as time varying broad band random noise whose characteristics are unknown and feedforward control strategy is used. For example, a car moves in varying speed through a road creates a non-stationary noise spectrum inside. The spectrum of the sound inside the compartment of a train, even if it moves with constant speed, changes when it passes through a tunnel or over a bridge. Hence, adaptive filters [6,7] are often used in ANC systems to tackle such situations. System identification view point of a single channel feed-forward ANCis system shown in figure 1 .

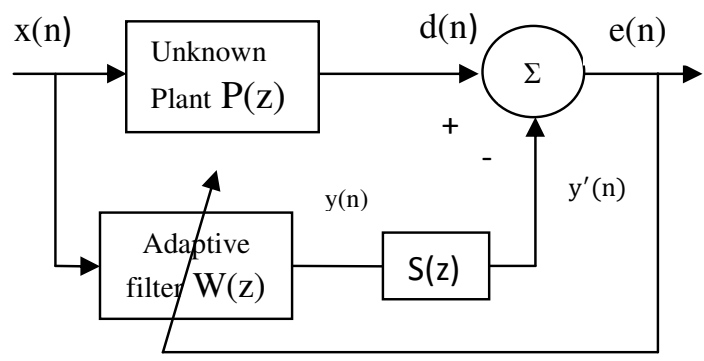

Figure 1. System identification view point of feed forward ANC

Here, $x(n)$ is the primary noise. $P(z)$ is the acoustic path whose characteristics are unknown. $\mathrm{d}(\mathrm{n})$ is the desired signal in adaptive control terminology. The summing junction of Figure 1 represents acoustic superposition in the space between the primary noise $x(n)$ and the secondary noise $y^{\prime}(n) . e(n)$ is the residual output or the error signal after destructive interference between the primary signal and the secondary signal. To have a perfect cancellation of the primary noise, it is necessary to compensate the secondary path transfer function $S(z)$ as shown in Figure 1, which contains D/A converter, reconstruction filter, power amplifier, loudspeaker, acoustic path from loudspeaker to error microphone. All these components of secondary path introduce signal delay and also add noise to the filter output which deteriorates system performance. Various methods were proposed in the past for tackling this problem [8] - [17]. In this paper, Filtered-X NLMS algorithm is used for estimating the secondary path transfer function.

\section{Proposed ANC System for a Noisy Speech}

An ANC system as shown in Figure 2 has been proposed for reducing noise from a noisy speech.Since the secondary path transfer function $S(z)$ follows the adaptive filter, conventional LMS algorithm must be modified to ensure convergence. Filtered-X LMS (FXLMS) algorithm [18] is one of the modified schemes popularly used for online estimation of secondary path transfer function. Here, instead of LMS algorithm, its variant NLMS algorithm is used for estimating secondary path transfer function. Hence the scheme may be called FXNLMS algorithm and the control filter updates using the equation

$$
\mathbf{w}(n+1)=\mathbf{w}(n)+\mu \mathbf{x}^{\prime}(n) e(n)
$$

Where, $\mathbf{w}(n)=\left[\begin{array}{llllll}w_{0}(n) & w_{1}(n) & \ldots & \ldots & w_{L-1}(n)\end{array}\right]^{T}$ is the vector of the coefficients of control filter at time $n, L$ is the order of the control filter, $\mathbf{x}^{\prime}(n)=\hat{s}(n) * \mathbf{x}(n), \hat{s}(n)$ is the estimated impulse response of the secondary-path filter $\hat{S}(z)$ and $\mathbf{x}(n)$ is the input vector.

The proposed system consists of two control filters $W_{1}(z) \& W_{3}(z)$ working in system identification configuration using NLMS algorithm. $P_{1}(z)$ and $P_{2}(z)$ are primary signal paths through acoustic medium whose characteristics are unknown and time varying. $x_{t}(n)$ is the actual noise signal collected from a passenger train cabin. Additive White Gaussian Noise 
(AWGN) has been added to it to incorporate uncertainty in the primary signal. $W_{2}(z)$ and $W_{4}(z)$ are adaptive filters used for estimating secondary path transfer functions $S_{1}(z)$ and $S_{2}(z)$ respectively. AWGN is used for estimating impulse responses [11] of secondary path transfer functions. A copy of the estimated secondary path transfer function $\hat{S}_{1}(z)$ is placed at the primary path and filtered primary signal is used for NLMS computation for estimating the coefficients of the control filter $W_{1}(z)$. Similarly a copy of the estimated secondary path transfer function $\hat{S}_{2}(z)$ is placed at the path of $e_{1}(n)$ and the filtered $e_{1}(n)$ signal is used for the computation of the coefficients of $W_{3}(z)$. The first adaptive control filter $W_{1}(z)$ when adaptively identical to $P_{1}(z)$, the error $e_{1}(n)$ is minimized. $e_{1}(n)$ is the primary signal for the second control filter $W_{3}(z)$. When $W_{3}(z)$ adaptively identical to $P_{2}(z)$, the error $e_{2}(n)$ will be further reduced.

The output of the system,

$$
Y_{o}(z)=E_{3}(z)
$$

where, $Y_{o}(z)$ is the z-transform of $y_{o}(n)$

$$
E_{3}(z)=P_{2}(z) E_{1}(z)-S_{2}(z) Y_{5}(z)+Z(z)_{L F}
$$

where, $Z(z)_{L F}$ is the z-transform of the low frequency content $(\leq 500 \mathrm{~Hz})$ of speech signal $z(n)$ and $S_{2}(z)$ is the secondary path transfer function associated with control filter $W_{3}(z)$. Again,

$$
\begin{aligned}
E_{3}(z) & =P_{2}(z) E_{1}(z)-S_{2}(z)\left\{W_{3}(z) E_{1}(z)+R(z)\right\}+Z(z)_{L F} \\
& =P_{2}(z) E_{1}(z)-S_{2}(z) W_{3}(z) E_{1}(z)-S_{2}(z) R(z)_{L F}+Z(z)_{L F} \\
& =\left[P_{2}(z)-S_{2}(z) W_{3}(z)\right] E_{1}(z)-S_{2}(z) R(z)_{L F}+Z(z)_{L F}
\end{aligned}
$$

Where $R(z)_{L F}$ is the z-transform of the low frequency content of AWGN.

Further,

$$
\begin{aligned}
E_{1}(z)= & X(z) P_{1}(z)-S_{1}(z) Y_{1}(z)+Z(z)_{L F} \\
& =X(z) P_{1}(z)-S_{1}(z)\left\{W_{1}(z) X(z)+R(z)_{L F}\right\}+Z(z)_{L F} \\
& =\left\{P_{1}(z)-S_{1}(z) W_{1}(z)\right\} X(z)+S_{1}(z) R(z)_{L F}+Z(z)_{L F}
\end{aligned}
$$

where, $S_{1}(z)$ is the secondary path transfer function associated with the control filter $W_{1}(z)$.

Substituting equation (5) in equation (4) we have

$$
\begin{gathered}
E_{3}(z)=\left[P_{2}(z)-S_{2}(z) W_{3}(z)\right]\left[\left\{P_{1}(z)-S_{1}(z) W_{1}(z)\right\} X(z)-S_{1}(z) R(z)_{L F}+Z(z)_{L F}\right]- \\
S_{2}(z) R(z)_{L F}+Z(z)_{L F}
\end{gathered}
$$

If error microphones for collecting $e_{1}(n)$ and $e_{3}(n)$ are kept nearby, the plant $P_{2}(z)$ may be considered unity as there will not be any uncertainty when signal travels very short distance in air. Then,

$$
\begin{gathered}
E_{3}(z)=\left[1-S_{2}(z) W_{3}(z)\right]\left[\left\{P_{1}(z)-S_{1}(z) W_{1}(z)\right\} X(z)-S_{1}(z) R(z)_{L F}+Z(z)_{L F}\right]- \\
S_{2}(z) R(z)_{L F}+Z(z)_{L F}
\end{gathered}
$$


Signal \& Image Processing : An International Journal (SIPIJ) Vol.3, No.3, June 2012

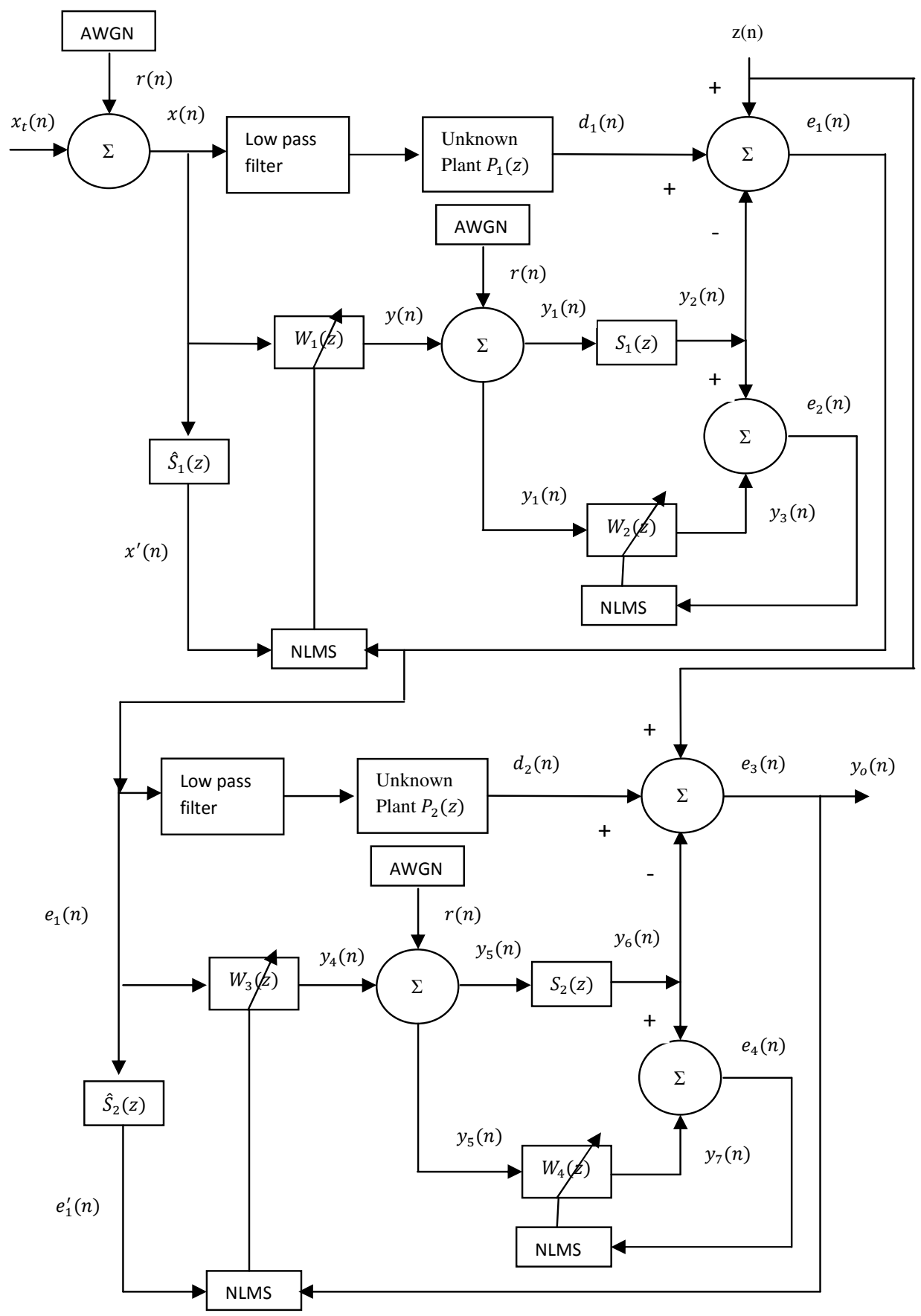

Figure 2. The proposed ANC system for noisy speech

When adaptive filter $W_{3}(z)$ converges, ideally $E_{2}(z)=0$ for low frequency signals. Then, from equation (7) we can have $Z(z)_{L F}=0, R(z)_{L F}=0$ and also

$$
1-S_{2}(z) W_{3}(z)=0
$$

From equation (8), it is evident that $S_{2}(z) W_{2}(z)=1$; which reveals that the secondary transfer function $S_{2}(z)$ has been compensated when the adaptive filter $W_{3}(z)$ converges. Hence at the 
output $y_{o}(n)$ contains speech signal compromised with very low frequency, added with very minimal residual noise.

\section{Simulation of the Proposed System}

The system as shown in figure 2 is simulated on a digital computer using matlab. Primary noise signal $x_{t}(n)$ is collected from an air-conditioned passenger train cabin for 5 seconds $(40,000$ samples). It is sampled at $8 \mathrm{KHz}$ and quantized to 16 bits/sample. As the primary path $P_{1}(z)$ is an acoustic medium and the characteristics of the path changes with time, random noise has been generated and added with $x_{t}(n)$. The resultant noise $x(n)$ is filtered through a low pass filter whose cut off frequency is $500 \mathrm{~Hz}$. . In this paper ANC is used for reducing low frequency noise. NLMS algorithm [1] is used for system adaptation.

The secondary path transfer functions $S_{1}(z)$ and $S_{2}(z)$ of the system are estimated online using adaptive filters $W_{2}(z)$ and $W_{4}(z)$ respectively. The estimated secondary path transfer functions are denoted as $\hat{S}_{1}(z)$ and $\hat{S}_{2}(z)$. Their impulse responses are estimated using cross correlation techniques by feeding AWGN signal to their inputs as shown in figure 2. The impulse responses of $\hat{S}_{1}(z)$ and $\hat{S}_{2}(z)$ are shown in figure 3 and 4 respectively. A copy of $\hat{S}_{1}(z)$ is placed in the primary signal path and its output is computed using the equation $x^{\prime}(n)=\hat{s}_{1}(n) * x(n)$; where, $\hat{S}_{1}(n)$ is the estimated impulse response of $S_{1}(z)$ and $x(n)$ is the primary signal. The symbol * denotes convolution. Similarly a copy of $\hat{S}_{2}(z)$ is placed in the path of $e_{1}(n)$. The error signal $e_{1}(n)$ is the primary signal for the control filter, $W_{3}(z)$. The output of the $\hat{S}_{2}(z)$ is computed by the equation $e_{1}^{\prime}(n)=\hat{s}_{2}(n) * e_{1}(n)$; where, $\hat{s}_{2}(n)$ is the impulse response of $\hat{S}_{2}(z)$.

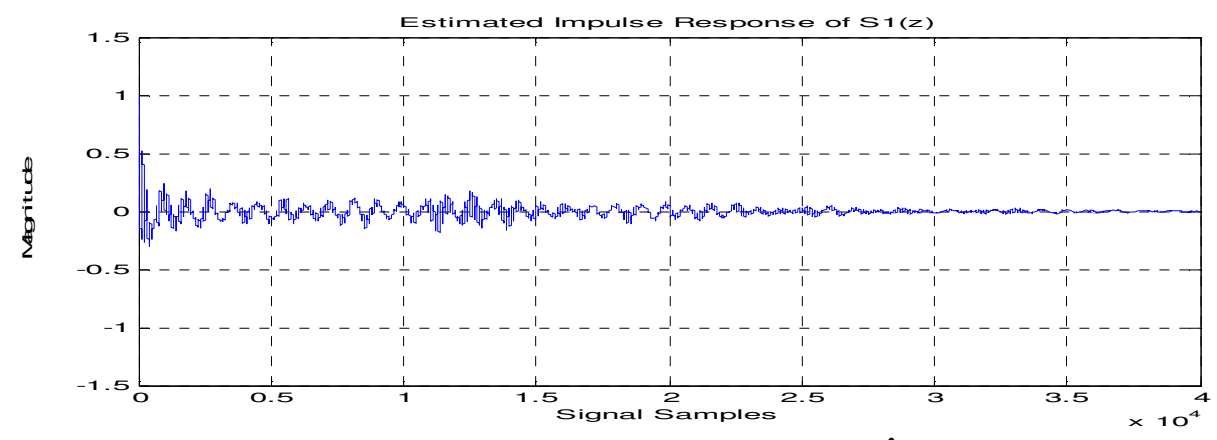

Figure 3. The impulse response of $\hat{S}_{1}(z)$

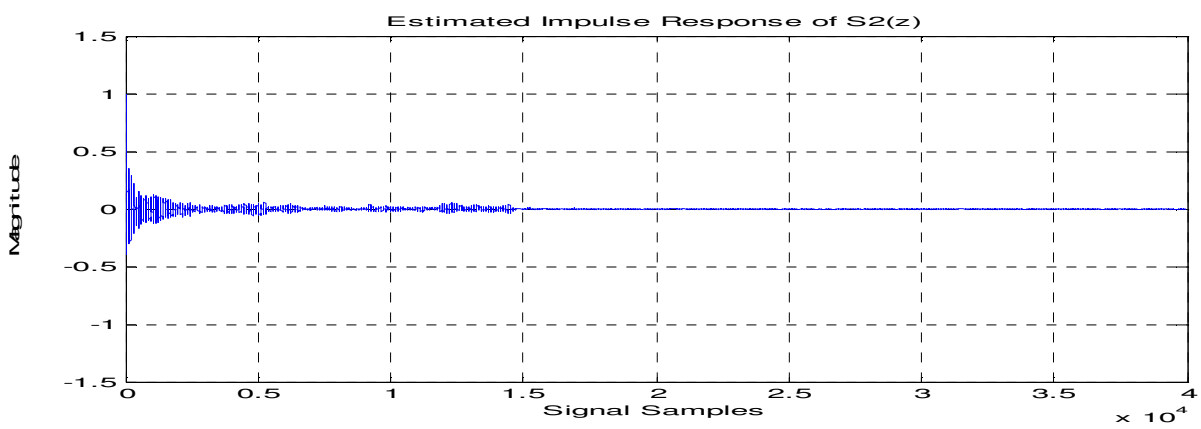

Figure 4. The impulse response of $\hat{S}_{2}(z)$

The adaptive filter $W_{1}(z)$ is tuned first by varying its step size $\mu_{1}$ from 0.01 to 3 with an increment of 0.01 and observed the minimum noise power of $e_{1}(n)$ as around $-7 \mathrm{~dB}$. The graph obtained by plotting $\mu_{1}$ versus $e_{1}(n)$ is depicted in figure 5 . The optimum $\mu_{1}$ of $W_{1}(z)$ is chosen 
from this curve as 1.5 for which it gives minimum power of $e_{1}(n)$ as -7.47 . The noise power of $e_{1}(n)$ is calculated using the formula $10 \log \left(\frac{\frac{\sum_{n=1}^{4000}\left(e_{1}(n)\right)^{2}}{40000} /}{\frac{\sum_{n=1}^{40000}\left(N_{S}(n)\right)^{2}}{40000}}\right)$; where $N_{S}(n)=$ $x(n)+z(n)$ is the noisy speech signal. $z(n)$ is the uncorrupted speech signal, "An icy wind racked the beach" which is selected from the Harvard list of phonetically balanced sentences. $x(n)$ is the filtered primary noise.

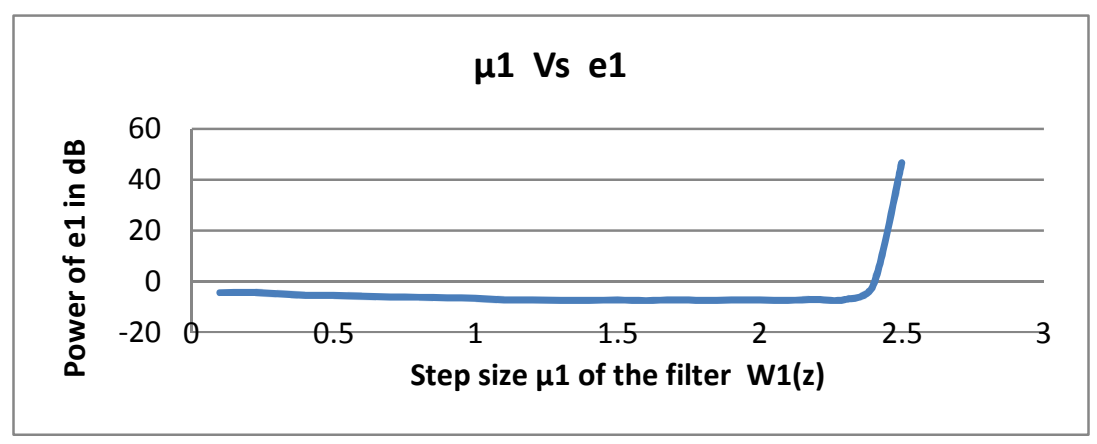

Figure 5. The curve of $\mu_{1}$ versus power of $e_{1}(n)$ in $\mathrm{dB}$

The adaptive filter $W_{3}(z)$ is tuned by varying the value of $\mu_{3}$ from 0.01 to 3 with an increment of 0.01 after fixing the value of $\mu_{1}$ as 1.5 and observed minimum residual noise power of $e_{3}(n)$ as around $-11 \mathrm{~dB}$. The graph obtained by plotting $\mu_{3}$ versus $e_{3}(n)$ is shown in figure 6 . The optimum value of $\mu_{3}$ of $W_{3}$ is chosen from this curve as 1.7 for minimum noise power $e_{3}(n)$.

The noise power $e_{3}(n)$ is calculated using the formula $10 \log \left(\frac{\sum_{n=1}^{40000}\left(e_{3}(n)\right)^{2}}{40000} / \frac{\sum_{n=1}^{4000}\left(N_{S}(n)\right)^{2}}{40000}\right)$.

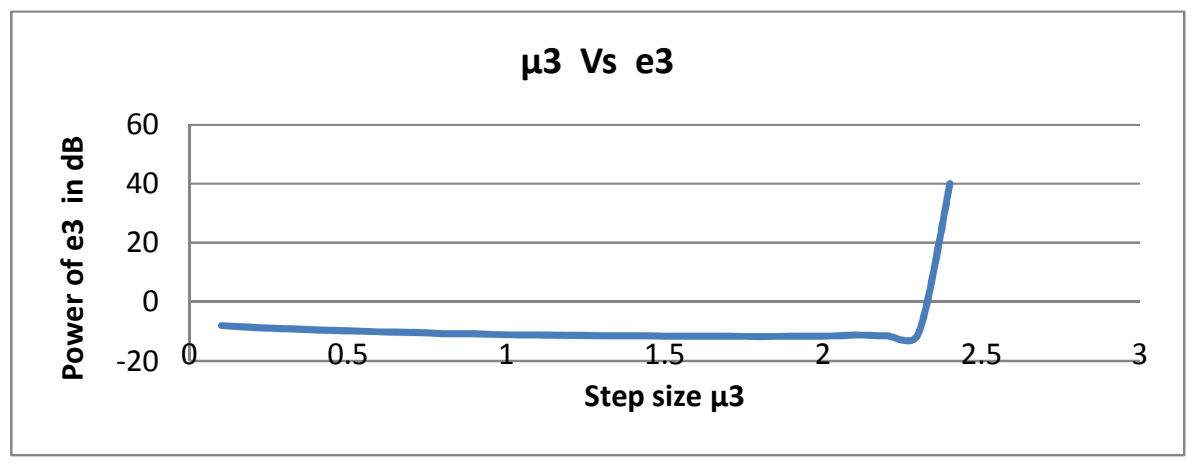

Figure 6. The curve of $\mu_{3}$ versus power of $e_{3}(n)$ in $\mathrm{dB}$

The values of $\mu_{1}$ and $\mu_{2}$ are fed in the simulation program and the proposed system is simulated with noisy speech. The error output $e_{3}(n)$ of the adaptive filter $W_{3}(n)$ is the final system output $y_{o}(n)$, which is the recovered speech signal. In this method, the low frequency noise from the noisy speech is reduced to the level of $-11 \mathrm{~dB}$. Uncorrupted speech signal $z(n)$ is 
Signal \& Image Processing : An International Journal (SIPIJ) Vol.3, No.3, June 2012

shown in figure 7. Noisy speech signal is depicted in figure 8 and system output $y_{o}(n)$ is shown in figure 9.

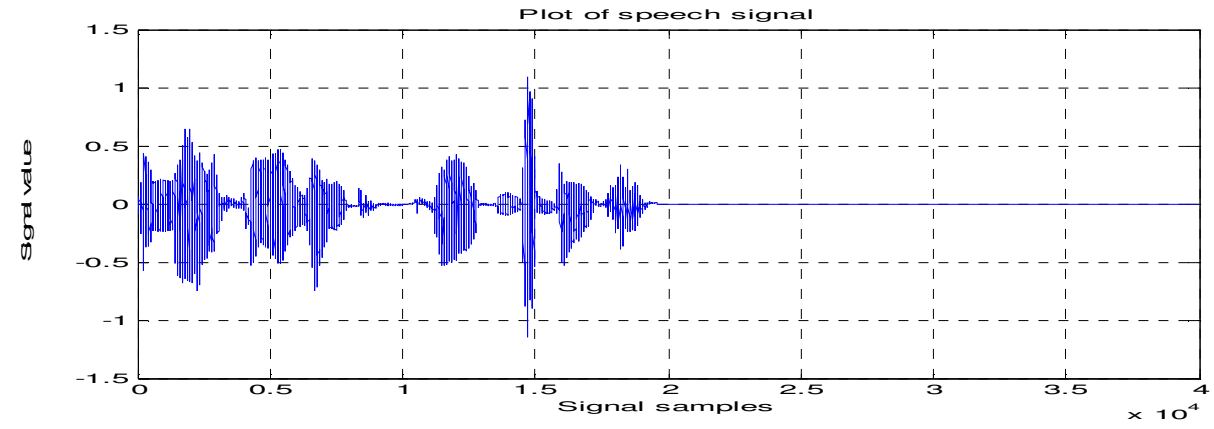

Figure 7. Uncorrupted speech signal 'An icy wind racked the beach'

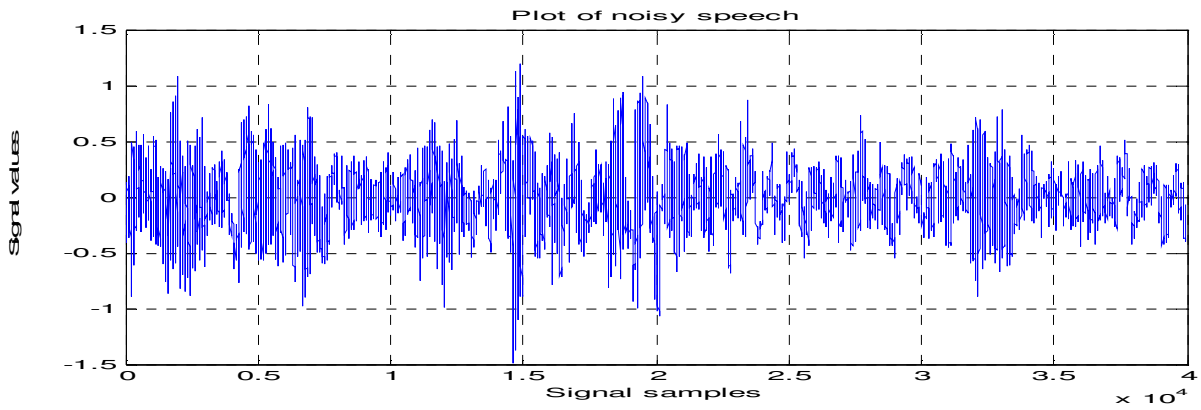

Figure 8. Noisy speech signal

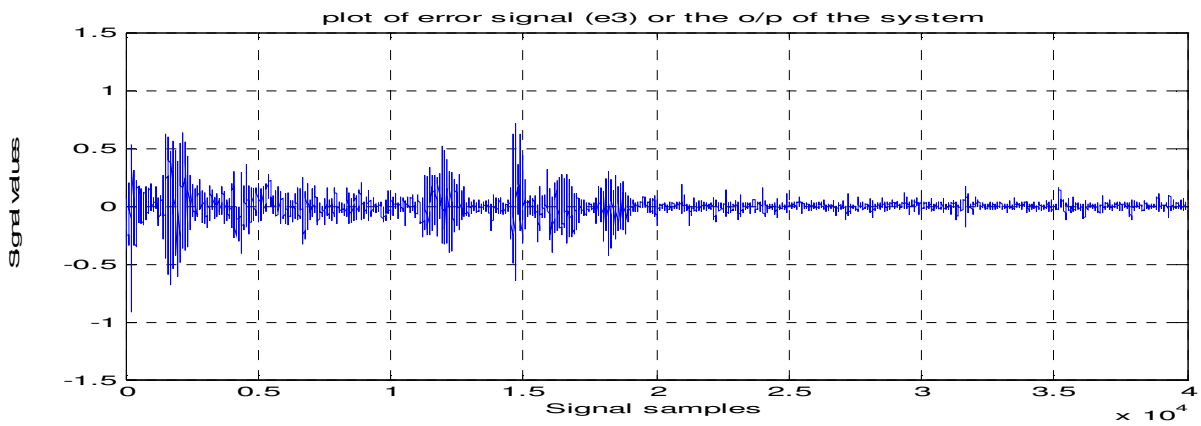

Figure 9 The recovered speech signal at the output of the system.

he intelligibility of the system output is tested using Mean Opinion Score (MOS) method. 25 listeners graded the system output on an absolute scale ranging between 1 and 5 ( 5-xcellent, 4good, 3-fair, 2-poor and 1-bad). The average quality of the intelligibility of the speech signal at the output is graded as 4 (good). 
Signal \& Image Processing : An International Journal (SIPIJ) Vol.3, No.3, June 2012

\section{Conclusion}

An Active noise control system is proposed for reducing the noise level from a noisy speech. The input to the system is a speech signal mixed with randomly varying noise collected from a passenger train cabin. The system is simulated with NLMS algorithm for updating filter coefficients. In this method the noise level of the corrupted signal is reduced to around $-11.6 \mathrm{~dB}$ and the intelligibility of the speech signal is found good. This kind of system can find application in mobile phones as mobile communication is common at market places and also in trains \& buses.

\section{REFERENCES}

[1] S.M. Kuo and D.R. Morgan,(1996) Active Noise Control Systems - Algorithms and DSP implementations, New Yourk: Wiley.

[2] C.H. Hansen and S.D. Snyder, (1997) Active Control of Noise and Vibration, London, U.K.; E\&FN.

[3] Sivadasan Kottayi and N.K. Narayanan, (2008)“"The technique of active noise control," Electronics for you, Vol. 40 No.6,pp 39-46

[4] Narayanan N.K. and Sivadasan Kottayi, (2011) "Active Noise Control”, Proceedings of the National Symposium on Acoustics, Jhansi, India, Nov. 17 - 19, 2011.

[5] Sivadasan Kottayi and N.K. Narayanan, (2010) "Active Noise Control: A simulation study", Proceedings of the International conference on Computer, Communication \& Intelligence, July 2223, Madurai, 2010, pp. 320-325.

[6] Simon Haykin, (1996) Adaptive Filter Theory, Prentice Hall, New Jersey, 1996.

[7] Sivadasan Kottayi \& N.K. Narayanan, "Adaptive filter and its applications ”, Special issue on International Conference on Computational Engineering Practices \& Techniques, Nov.25-26, 2010, Kuttipuram, India, MES Journal of Technology \& Management Vol.1, No. 02, pp 149-161.

[8] D.R. Morgan, (1980) "An analysis of multiple correlation cancellation loops with a filter in the auxiliary path,” IEEE Trans. Acoust., speech, Signal Processing, Vol. ASSP-28, 454-467.

[9] J. C. Burgess, (1992) “Chirp dsign for acoustical system identification," Journal of Acoustical Society of America, 91 (3), pp 1525-1530.

[10] B. Widrow and S.D. Stearns, (1985) “Adaptive Signal Processing,” Englewood Cliffs, N.J., PrenticeHall.

[11] L.J. Eriksson and M.C. Allie, (1989) "Use of random noise for on-line transducer modeling in an adaptive active attenuation system," Journal of Acoust. Soc. America, vol. 85, pp 794-802.

[12] C. Bao, P. Sas and H. V. Brussel, (1993) "Comparison of two online identification algorithm for active noise control," Proc. Recent Advances in Active Control of Sound Vibration, 1993, pp. 38 51.

[13] C. Bao, P. Sas and H. V. Brussel,(1993) "Adaptive active control of noise in 3-D reverberant enclosures," J. Sound Vibr., vol. 161, pp $501-514$.

[14] S.M. Kuo and D.Vijayan, (1997) "A Secondary path modeling technique for active noise control systems", IEEE Transactions on Speech and Audio Processing, vol. 5. No.4, pp 374-377.

[15] M. Zhang, H. Lan and W.Ser, (2001) "Cross-updated active noise control system with online secondary path modeling”, IEEE Transactions on speech and audio processing, vol. 9, No. 5, pp 598602.

[16] M.T. Akhtar, M. Abe and M. Kawamata, (2006) “ A new variable step size LMS algorithm based method for improved online secondary path modeling in Active Noise Control Systems," IEEE Transaction on Audio, Speech and Language Process, vol.14, No. 2, 720-726. 
Signal \& Image Processing : An International Journal (SIPIJ) Vol.3, No.3, June 2012

[17] A. Carini and S. Malatini, (2008) "Optimal variable step-size NLMS algorithms with auxiliary noise power scheduling for feedforward active noise control”, IEEE Trans. Audio, Speech and Lang Process., vol. 16, no. 8, 1383-1395.

[18] B.Widrow, D.Shur and S. Shaffer, "On adaptive inverse control”, Proc. $15^{\text {th }}$ Asilomar Conf., 1981, pp 185-189.

\section{Authors}

\section{SIVADASAN KOTTAYI}

Sivadasan Kottayi had his M.Tech (Electronics \& Communication Systems) from CUSAT, Kerala, India in 1981. He served BITS, Pilani; CEERI, Pilani; NERIST, Itanagar; JNAU, Jabalpur; Defence University, Ethiopia; Arbaminch University, Ethiopa in different capacities. Currently he is working as a faculty member of EIET Department of Yanbu Industrial College, Saudi Arabia. He had to his credit two patents and many research publications in national and international Journals in the area of Instrumentation, Industrial Process Control, Microprocessor/microcontroller applications and Digital Signal Processing.

\section{N. K. NARAYANAN}

Dr. N.K.Narayanan is currently working as Professor of the School of Information Science \& Technology, Kannur University, Kerala, India. He served on deputation as Principal, Co-operative Engineering College, Vadakara, Kerala, India from April 2008 to March 2010. He earned a Ph.D in speech signal processing from Department of Electronics, CUSAT, Kerala, India in 1990. He has published more than seventy research papers in national \& international journals in the area of Speech processing, Image processing and Neural network.

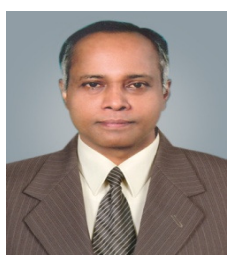

